\title{
Contribution of Elecromyographic Examination to Neuro-Occlusal Rehabilitation
}

\author{
Amel Belkhiri* \\ University Hospital Center Blida, Algeria \\ *Corresponding author: Amel Belkhiri, University Hospital Center Blida, Algeria, Email: amelbelkhiri2@gmail.com
}

Submission: 非 May 24,2018; Published: 㘹June 07, 2018

\begin{abstract}
For Pedro Planas, the indirect tracks allow, in addition to the treatment of certain malocclusions, to release laterality movements and thus increase the activity of the masticatory muscles. The objective of this work will be to observe the effect of the Planas device on the activity of the masseter and temporal anterior, on each side, during right and left lateral movements. An electromyogram is used to assess the bioelectrical activity of these muscles with and without tracks in place.
\end{abstract}

\section{Introduction}

The chewing function participates fully in the development of maxillary only under the condition that it is done in right-left alternative laterality. Studies have shown that patients with malocclusions tend to have pathological chewing. Pedro Planas proposes its indirect tracks which allow in addition to the treatment of certain malocclusions to release the movements of laterality and thus increase the activity of the masticatory muscles. Although this movement of laterality is not functional in itself, it is however the one that best imitates the functional masticatory situation: it is the movement that the clinician must examine to study the patient's chewing. The aim of this work is to observe the effect of the Planas device on the activity of the anterior masseters and temporals, on each side, during right and left lateral movements. An electromyogram was used to assess the bioelectrical activity of these muscles [1-3].

\section{Materials and Methods}

Fifteen children aged between 7 and 10 years were selected in orthodontic service of the university hospital center of Blida. All of patients show malocclusions and follow a neuro-occlusal rehabilitation treatment, using Planas indirect tracks. Nine patients have class I malocclusions, five suffer from mandibular retrognathia and one patient shows skeletal class IIII reports. Patients were evaluated during orthodontic treatment, 3-4 months after placement of the Planastracks (Figure 1).
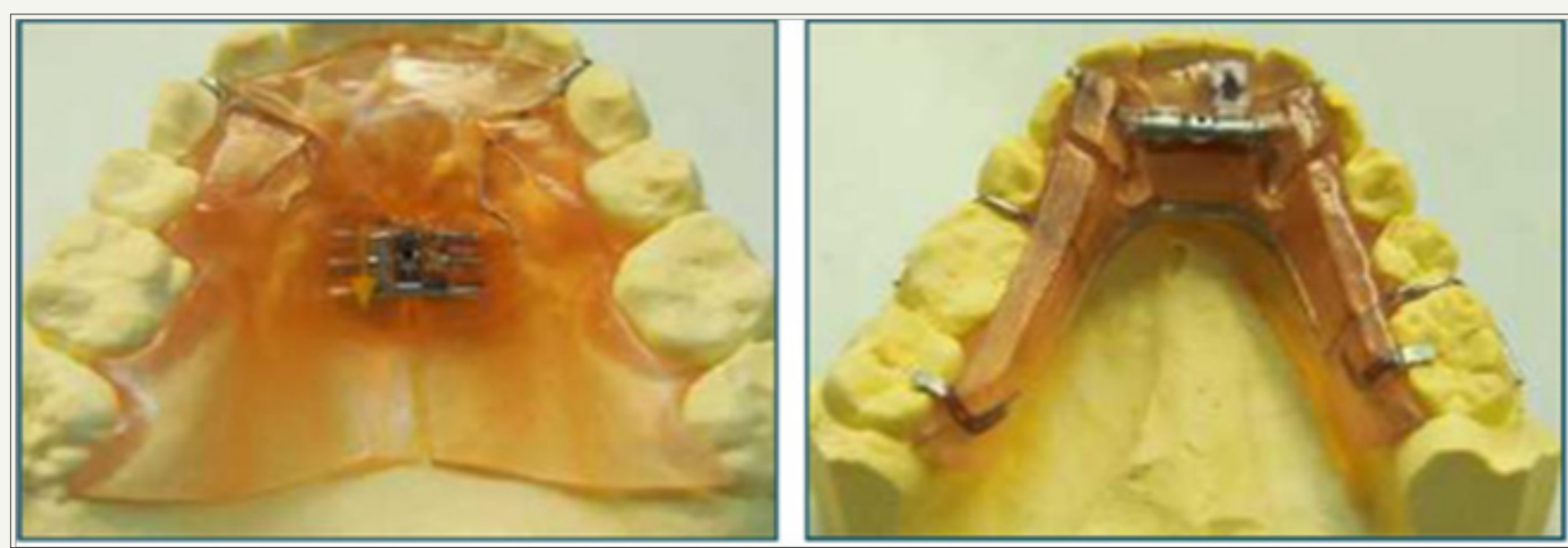

Figure 1: Plates with tracks or indirect tracks of Planas. 
The control group is represented by the same patients, without the Planas plates. The electromyogram used for this work is a neuro bipolar EMG eb with:
1) Two dynamic recording channels and a frequency response of $20 \mathrm{KHz}$ during a 100 millisecond scan;

2) Integrated acoustic and visual stimulators;

Figure 2: Neuro bipolar EMG eb.

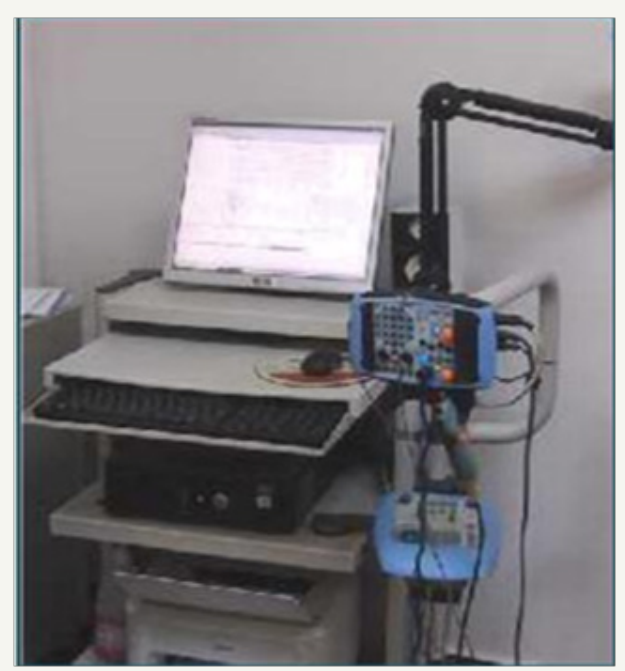

3) Computer including all necessary software, hardware and accessories (Figure 2).

The graphical recording of the anterior masseter and temporal muscles on both sides for each patient was obtained by means of self-adhesive electrodes fixed on the skin covering the muscles concerned, following the direction of the muscle fibers detected by palpation. The impedance at the passage of the current was reduced by a scrupulous cleaning of the cutaneous part used, with alcohol at $90^{\circ}$. To obtain these recordings, each patient was asked to remove his orthodontic appliance and perform right and then left lateral movements (Figure 3).

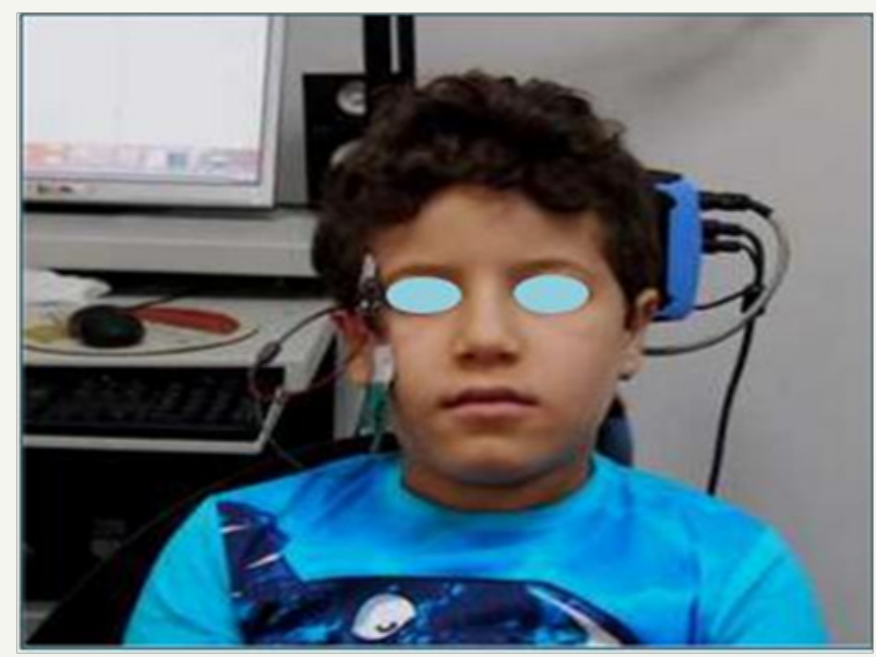

Figure 3: Self-adhesive electrodes.

The same procedure was carried out for the recording of the electrical activity of the temporals and masseter during laterality movements, with the Planas apparatus installed. For all recordings, the patient was seated, with the Frankfurt plane almost parallel to the ground. In addition, efforts have been made to create an atmosphere of tranquility and silence. All recordings were made by the same neurologist.

\section{Results}

The computer provides a qualitative record of the frequency spectrum achieved by the patient's muscles.

The record obtained corresponds to the maximum power developed by the muscular system. In each patient who performs plate-free slippery laterality movements, a moderate increase in 
activity is observed for all recorded muscle groups. It is also noted that the electromyographic activity of these same muscle groups, with the plates installed, is higher for 14 out of 15 patients. The bioelectric potential for the anterior temporal muscle on the displacement side is greater than that of the masseter (Figure 4-7).

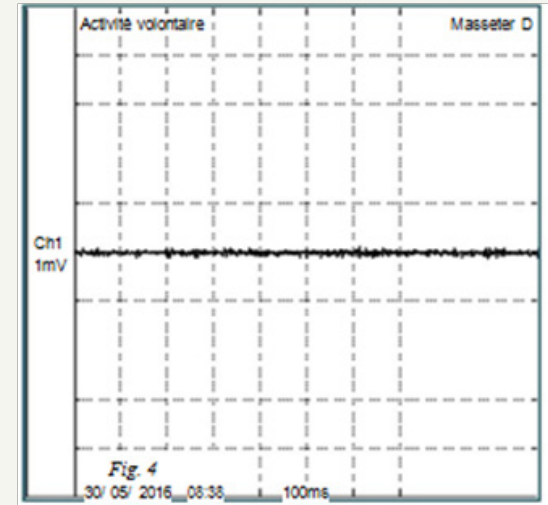

Graphical activity of the right masseter Without the Planas tracks.

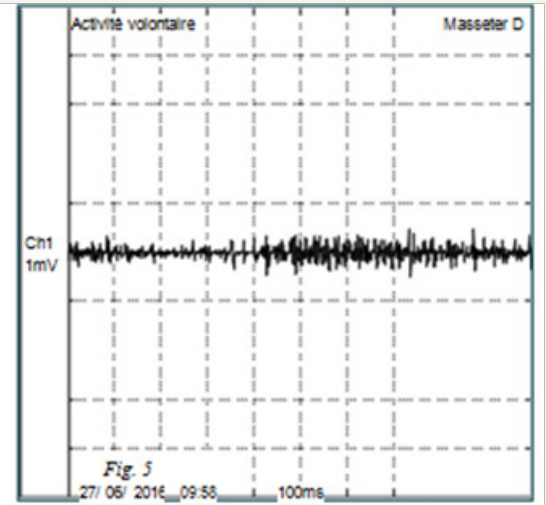

Graphical activity of the right masseter

With the Planas tracks.

Figure 4: Graphic recording of the right masseter without and with Planas tracks.

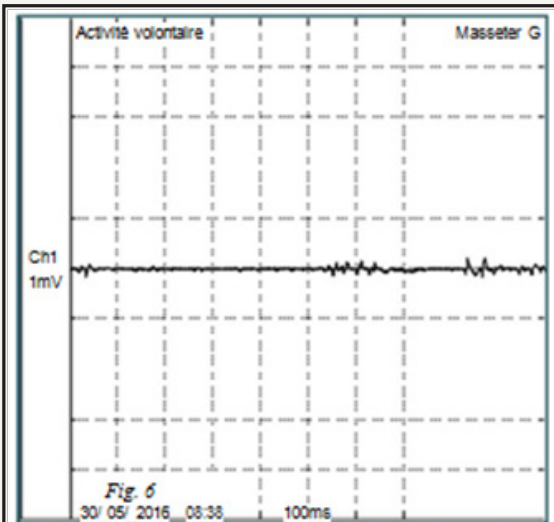

Graphical activity of the left masseter Without the Planas tracks.

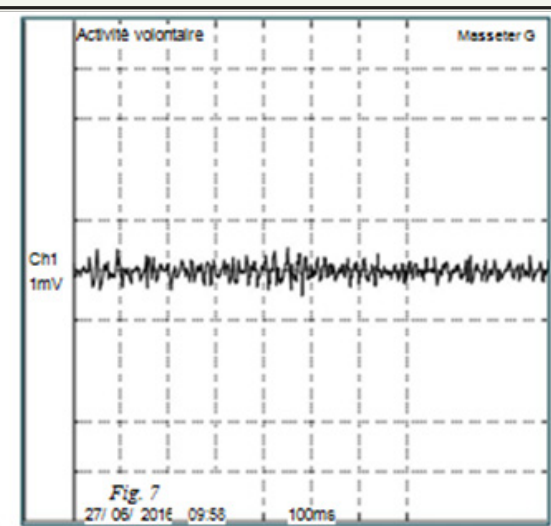

Graphical activity of the left masseter

With the Planas tracks.

Figure 5: Graphic recording of the left masseter without and with Planas tracks.

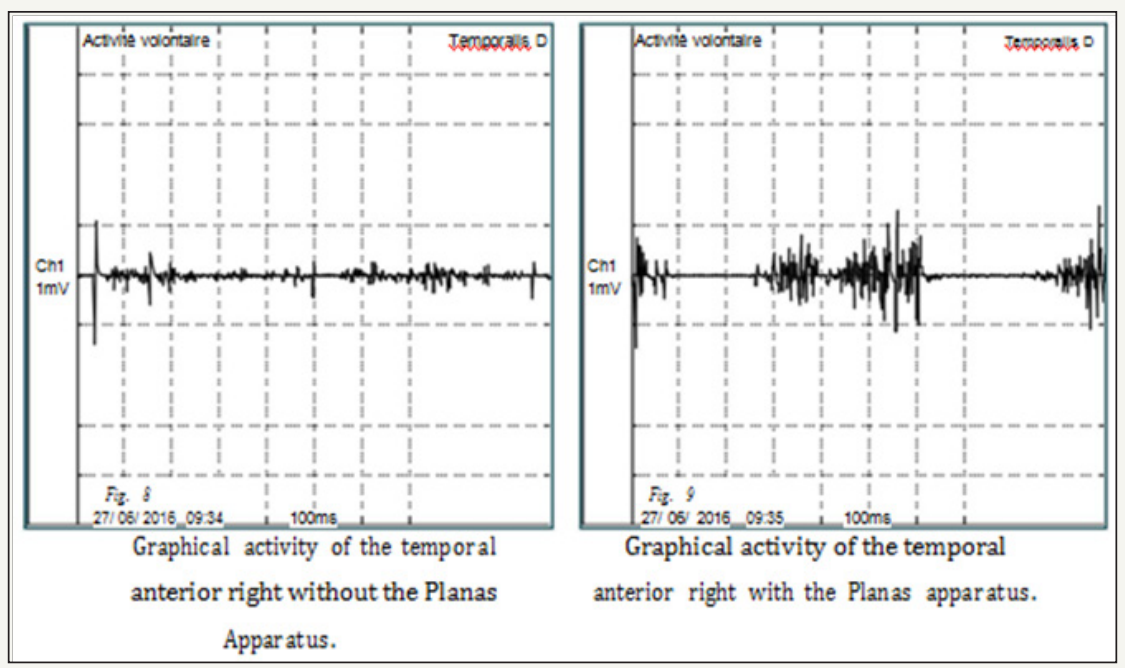

Figure 6: Graphical recording of the temporal anterior right without and with device of Planas. 


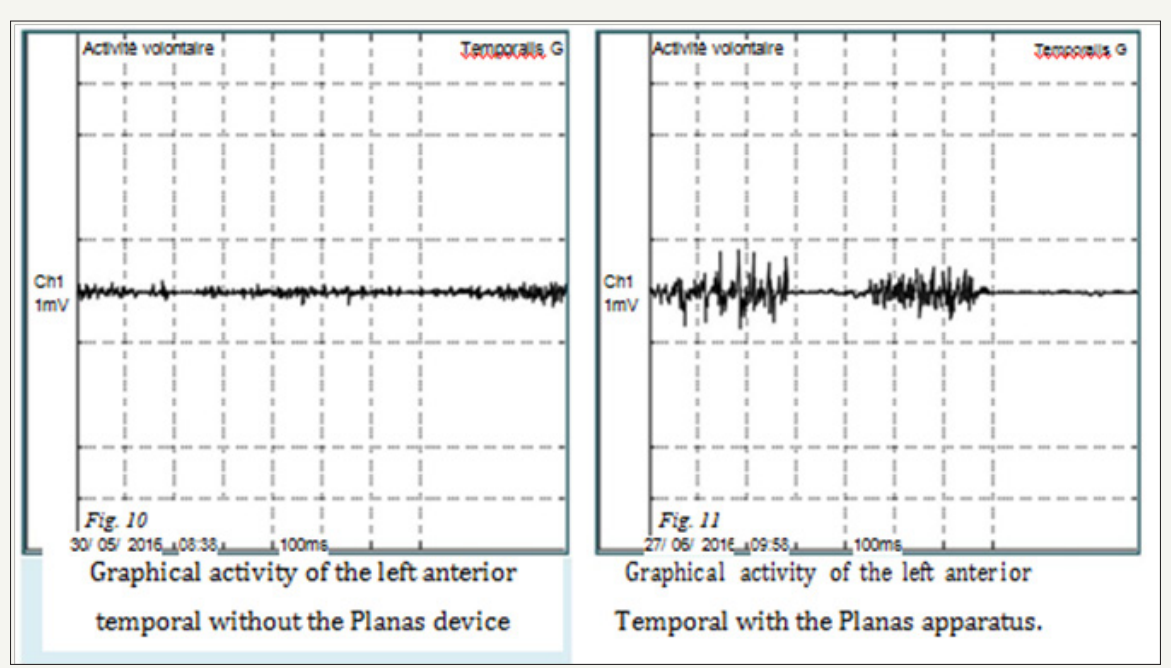

Figure 7: Graphical recording of the anterior temporal left without and with Planas tracks.

\section{Discussion}

Electromyography is the recording of muscle action currents. Indeed, muscle contraction is accompanied by an electrical phenomenon that can be recorded. When the muscular fibers receive with a sufficient intensity a nerve impulse coming from the motor neuron, a certain number of electrical modifications take place. This sudden modification of the polarization determines the action current that is recorded and is reflected on an electromyographic trace. When the contraction is light, it is accompanied by the activity of a limited number of motor units (neuron and muscle fibers to which the terminal arborizations of this neuron are distributed) and the EMG recording is relatively simple [4-7]. The contraction increases with the intensity of the stimulus by recruitment of new motor units. The currents of action are more numerous, they are superimposed and the amplitude of the plot increases. In this work, the stimulation was induced by the Planas track plates, which freed the patient from any blockage or constraint during his laterality movements. Motor unit recruitment was not similar in all subjects in our study. The myoelectric potential was significantly lower for the anterior temporal muscles and the masseters in one of the 15 patients, while having the device in place. This is because the latter only occasionally wore his orthodontic device. In his study Santana Penin found an increase in the activity of the muscles studied in 100 percent of the cases.

\section{Conclusion}

The activity of the temporal and masseter muscles during sliding laterality movements is greater when the Planas tracks plates are laid. It can be concluded that these plates allow the patient to more easily perform right and left mandibular lateral movements, which then begins to "exercise with his masticatory muscles". These muscular stresses are transmitted via the device to the dental and mucosal structures and thus to the periodontal, bone and sutural tissues that constitute the growth sites of the maxillomandibular complex. When established malocclusions exist, treatment with Planas track plates can be used to stimulate the development of the masticatory apparatus, thus breaking the vicious circle; hypo-function-under development.

\section{References}

1. Ahlgren J, Sonesson B, Blitz M (1985) An elecromyographic analysis of normal occlusion. Am J Orthod 87(3): 230-239.

2. Kolf J (2002) Apropos de la mastication. Rev d'Orthop Dento-Faciale 36: 7-10.

3. Martín C, Palma JC (2012) Longitudinal evaluation of sEMG of masticatory muscles and kinematics of mandible changes in children treated for unilateral cross-bite. J Electromyogr Kinesiol 22(4): 620-628.

4. Planas P (1991) Notre testament en RNO. Orthod Fr 62: 695-708.

5. Planas P (2006) Réhabilitation neuro-occlusale, CDP, Paris, France.

6. Santana Penin UA (1992) Anomalies d'inclinaison du plan occlusal: traitement précoce par les pistes indirectes-Réponse électromyographique Orthod Fr 63(2): 619-633.

7. Stohler CS (1986) A comparative elecromyographic and kinesiographic study of deliberate and habitual mastication. Arch Oral Biol 31(10): 669678. 
Creative Commons Attribution 4.0 International License

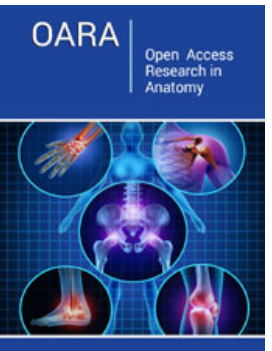

\section{Open Access Research in Anatomy}

\section{Benefits of Publishing with us}

- High-level peer review and editorial services

- Freely accessible online immediately upon publication

- Authors retain the copyright to their work

- Licensing it under a Creative Commons license

- Visibility through different online platforms 